\title{
Erratum to: Synthesis of Macrocyclic Depsipeptides via the Passerini-Click Reaction Strategy
}

\author{
I. V. Kutovaya ${ }^{a}$ and V. G. Nenajdenko ${ }^{a, *}$ \\ ${ }^{a}$ Faculty of Chemistry, Moscow State University, Moscow, 119991 Russia \\ *e-mail:nenajdenko@gmail.com
}

Received November 10, 2020; revised November 10, 2020; accepted November 10, 2020

DOI: $10.1134 / \mathrm{S} 1070428020100334$

The article I.V. Kutovaya and V.G. Nenajdenko "Synthesis of Macrocyclic Depsipeptides via the Passerini Click Reaction Strategy" has no funding support by grant no. 18-53-34002 or any other grant.

The original article can be found online at https://doi.org/10.1134/S1070428020040016 